\title{
Effects of Calcium Content and Homogenization Method on the Microstructure, Rheology, and Stability of Emulsions Prepared with Soybean Flour Dispersions
}

\author{
Andrés L. Márquez,* Jorge R. Wagner, and Gonzalo G. Palazolo
}

The objective is to study the microstructure, rheology, and quiescent stability of oil-in-water emulsions prepared with defatted soybean flour dispersions, analyzing the effects of calcium concentration and homogenization method. Dispersions are prepared without or with addition of calcium chloride at different concentrations $\left(30,60\right.$, and $\left.90 \mathrm{mg} \mathrm{Ca} 100 \mathrm{~g}^{-1}\right)$ and treated by heating $\left(100{ }^{\circ} \mathrm{C}\right.$, $40 \mathrm{~min}$ ) and high-pressure homogenization ( $\mathrm{HPH}$ ) at $120 \mathrm{MPa}$. Emulsions are prepared with homogenized dispersions and sunflower oil by different homogenization methods (high-speed homogenization, $\mathrm{HSH}$; and $\mathrm{HPH}$ at 10 and $20 \mathrm{MPa}$ ). The addition of calcium produces a decrease of soluble protein in dispersions, leading to emulsions with higher oil droplet size and aggregation of particles when $\mathrm{HSH}$ and $\mathrm{HPH}$ are applied, respectively. The highest aggregation degree is observed in the systems with $30 \mathrm{mg} \mathrm{Ca} 100 \mathrm{~g}^{-1}$, attributed to the formation of a hydrated network of aggregated particles (oil droplets, protein aggregates, and insoluble fibers). Consequently, these emulsions also show the highest viscosity and creaming stability. The findings indicate that calcium can act as a functional ingredient in food emulsions prepared with soybean flour dispersion, because the texture and stability of the system can be controlled by the variation of calcium concentration and homogenization method.

Practical Applications: In this study, the impact of calcium addition and homogenization method on the microstructure, rheological behavior, and quiescent stability of o/w emulsions prepared with soybean flour dispersions is assessed. Unlike other works, the strategy used for calcium addition is to promote the interaction of the mineral with proteins and non-starch polysaccharides present in soybean flour and then reduce the size of aggregates by high-pressure homogenization. The mentioned strategy can allow the obtaining of vegetable calcium-rich emulsion-based products with texture and consistency modulated by the amount of added calcium and the applied homogenization method.

\section{Introduction}

Defatted soybean flour (DSF) is obtained as a by-product from the extraction of soybean oil and constitutes a rich source of protein and dietary fiber. The high protein content of DSF also opens the door to its potential use for the formulation of food emulsions, though the presence of dietary fiber may have a negative effect on its emulsifying properties. ${ }^{[1]}$ Nevertheless, the inclusion of dietary fiber in the diet, as non-starch polysaccharides, is believed to be important for optimum health. These components show various positive physiological effects in the small and large intestine. ${ }^{[2]}$ Moreover, it has been well accepted that calcium is essential for the structure and function of bone metabolism, for muscle functions, blood pressure, bone density, coagulation process, and releasing neurotransmitters. ${ }^{[3]}$ Yet, most of the worldwide population does not satisfy their calcium needs. Hence, calcium supplementation of food is a worldwide health challenge. ${ }^{[4]}$ Calcium fortification is usually related to fluid soybean products such as soymilk, because bovine milk presents a considerably higher content of that mineral. ${ }^{[5]}$ Furthermore, it is widely known that divalent cations tend to bind by the storage soy proteins (glycinin and $\beta$-conglycinin), phytate, and phospholipids, inducing aggregation and destabilization of the system. ${ }^{[6-9]}$ Indeed, by isothermal titration calorimetry studies, the water molecule release, either from the hydration shells of the calcium ion and/

Dr. A. L. Márquez, Dr. J. R. Wagner, Dr. G. G. Palazolo

Laboratorio de Investigación en Funcionalidad y Tecnología de Alimentos (LIFTA), Departamento de Ciencia y Tecnología,

Universidad Nacional de Quilmes, Roque Sáenz Peña 352 (B1876BXD)

Bernal, Buenos Aires, Argentina

E-mail: almarquez@conicet.gov.ar

Dr. A. L. Márquez, Dr. J. R. Wagner, Dr. G. G. Palazolo

Consejo Nacional de Investigaciones Científicas y Técnicas (CONICET), (C1425FQB)

Ciudad Autónoma de Buenos Aires, Argentina

DOI: 10.1002/ejlt.201700500 or dehydration of the hydrophobic core of the proteins, may be the driving energy source for binding of calcium by soy proteins, instead of Coulomb interaction. ${ }^{[10]}$ In addition, previous studies concerned with the calcium fortification of enteral formulas containing dietary fiber suggest that the divalent cation also interacts with soy polysaccharides. ${ }^{[1]}$

It should also be considered that the application of a thermal treatment is required to inactivate enzymes, such as lipoxygenase and antinutritional factors (Kunitz trypsin inhibitor and lectin) in soy-based protein products. Although heating is a necessary step to improve the nutritional value and taste of 
soymilk, when it is performed in the presence of calcium salts the insolubilization by aggregation of storage soy proteins and phytate is highly promoted. ${ }^{[7,12]}$

Different methods have been attempted in order to enhance the stability of calcium-fortified soybean products, usually pointing to avoid the interaction of the divalent cation with proteins and phospholipids. Previous works showed that it is possible to obtain a heat-stable soymilk fortified with calcium by the addition of citrate and phosphate as sequestering agents. ${ }^{[13-15]}$ Double emulsions were used as a medium to isolate calcium in the inner aqueous phase from soybean components in the outer aqueous phase. ${ }^{[16]}$ The results reported in previous works show that it is also possible the calcium fortification of soy beverages in the presence of dietary fiber. Krongsin et al. ${ }^{[4]}$ found that the addition of isolate fiber sources, such as pomelo pectin, enhanced the stability of calcium-fortified, acidified soymilk without addition of chelating agents. Recently, we have formulated a stable soybean beverage with addition of calcium salts at relatively high levels, following a novel strategy: firstly promoting the interaction of the divalent cation with proteins and fiber during a thermal treatment; and then homogenizing the destabilized system in order to achieve the reduction of the size of the formed aggregates. ${ }^{[17]}$ In the present study, we proposed the preparation of DSF aqueous dispersions with addition of calcium chloride at different concentrations using a similar strategy. The resultant dispersions were used as the continuous aqueous phase of emulsions prepared by different homogenization methods.

Therefore, the objective of this work was to study the effects of calcium concentration and homogenization method on the microstructure, rheology, and quiescent stability of oil-in-water (o/w) emulsions prepared with DSF dispersions and sunflower oil as lipid phase. Unlike the previous work, ${ }^{[17]}$ the lipid content of emulsions was substantially higher. In this way, the potentiality of a calcium-fortified food emulsion including soybean proteins and fibers was analyzed.

\section{Experimental Section}

\subsection{Materials}

Active defatted soy flakes were provided by Bunge Argentina S.A. (Puerto General San Martín, Santa Fe, Argentina). They were provided without thermal inactivation of antitryptic factors; moreover, storage soy proteins kept their native state. ${ }^{[18]}$ The flakes were ground in all purpose smashing machine (Chincan, FW Model; China) and the resultant flour was subsequent sieved at 500 (ASTM E-11-81, Zonytest; Buenos Aires, Argentina) and $125 \mu \mathrm{m}$ (ASTM E-11-70, Zonytest; Buenos Aires, Argentina), so that $95 \%$ of the initial sample passed through both sieves. DSF sample was finally obtained; its proximate composition (g/100 g, as dry basis) was crude protein, $55.1 \pm 0.4(\mathrm{~N} \times 6.25)$; ash, $7.7 \pm 0.1$; insoluble dietary fiber, $20.6 \pm 0.6$; soluble dietary fiber, $3.3 \pm 0.2$; galacto-oligosaccharides, $10.0 \pm 0.2$; and lipids, $1.9 \pm 0.1$. The moisture content of

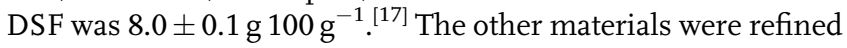
sunflower oil (Molino Cañuelas SACIFIA; Cañuelas, Argentina);

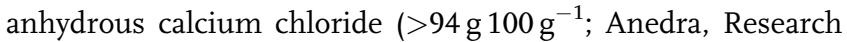
AG; Talar, Argentina); bovine serum albumin (>99 g/100 g, fatty acid free; Sigma Chemical Co.; St. Louis, MO, USA). Distilled water was used in all assays.

\subsection{Preparation of Aqueous Dispersions}

DSF was dispersed in distilled water under magnetic stirring for at least $2 \mathrm{~h}$. The dispersion contained $7.5 \mathrm{~g}$ total solids per 100 , which corresponds to $8.2 \mathrm{~g}$ DSF $100 \mathrm{~g}^{-1}$. Calcium chloride was added in order to obtain dispersions with 30, 60, and $90 \mathrm{mg} \mathrm{Ca} 100 \mathrm{~g}^{-1}$ (C30, C60, and C90, respectively), without considering the proper calcium content of DSF. An aqueous dispersion without added calcium (C0) was prepared as control sample. All dispersions were heated in a boiling water bath for $40 \mathrm{~min}$ and then cooled to room temperature $\left(\approx 25^{\circ} \mathrm{C}\right)$, producing the required inactivation of anti-nutritional factors and lipoxygenase and promoting calcium-storage protein/fiber interactions. $^{[11,12,17]}$ The cooked dispersions were pre-homogenized using an Ultra-Turrax T-25 high-speed rotor/stator device with a S25-20NK-18G dispersing tool (IKA-Labortechnik; Staufen, Germany) at $24000 \mathrm{rpm}$ for $1 \mathrm{~min}$. Finally, each dispersion was recirculated three times through a twin-stage valve high-pressure homogenizer (Panda 2 K, GEA Niro Soavi; Parma, Italy) in order to homogenize the aggregated system; the homogenization pressure was 120 and $12 \mathrm{MPa}$ in the first and second valves, respectively. The $\mathrm{pH}$ values of aqueous dispersions were determined using a $\mathrm{pH}$ meter (Sartorius PYP10-2S electrode; Göttingen, Germany); a two-point calibration (pH 4.0 and pH 7.0 standard buffer solutions) was performed.

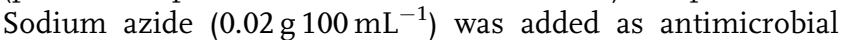
agent. Homogenized dispersions were used as the continuous aqueous phase of emulsions. Dispersions were prepared in duplicate.

\subsection{Preparation of Emulsions}

Each homogenized dispersion was mixed with sunflower oil in order to prepare o/w emulsions with $20 \mathrm{~g}$ lipid phase per $100 \mathrm{~g}$. Emulsions were prepared by three homogenization methods: high-speed homogenization, using the rotor/stator device (S25-20NK-18G dispersing tool) at $24000 \mathrm{rpm}$ for 1 min (HS treatment); high-speed homogenization plus high-pressure homogenization, using the valve homogenizer at 10.0 and $1.0 \mathrm{MPa}$ in the first and second valves, respectively (HP1 treatment); and high-speed homogenization plus high-pressure homogenization at 20.0 and $2.0 \mathrm{MPa}$ in the first and second valves, respectively (HP2 treatment). In this way, emulsions with different homogenization methods (HS, HP1, and HP2) and different added calcium concentrations in the aqueous phase $(0,30,60$, and $90 \mathrm{mg} 100 \mathrm{~g}^{-1}$ ) were obtained. Thus, emulsions were named according to the combination of homogenization method and calcium concentration, as follows: HS-0, HS-30, HS-60, HS-90, HP1-0, HP1-30, HP1-60, HP1-90, HP2-0, HP2-30, HP2-60, and HP2-90. Emulsions were prepared in duplicate and stored at $7^{\circ} \mathrm{C}$ for 1 week. 


\subsection{Particle Size Distribution (PSD)}

PSD of fresh dispersions and emulsions was determined by laser diffraction using a Malvern Mastersizer 2000E analyzer (Malvern Instruments Ltd.; Worcestershire, UK). PSDs were expressed as surface frequency $(\times 100)$. The refractive indices applied were 1.33 for dispersant, 1.52 for dispersion particles, and 1.47 for emulsion particles; and the absorption coefficients were 0.1 for dispersion particles and 0.001 for emulsion particles. The De Brouckere, volume-weighted, moment mean diameter $\left(D_{4,3}\right)$ was obtained from the PSD; this parameter was used in order to enhance the contribution of larger particles to the mean diameter and determine the relative aggregation degree (i.e., aggregates size) in emulsions. Samples were diluted in water in the dispersion system (Hydro 2000MU, Malvern Instruments Ltd.) at a speed of $2000 \mathrm{rpm}$. Emulsions were also analyzed after storage at $7^{\circ} \mathrm{C}$ for 7 days, carefully mixing the samples to redisperse the cream phase. Measurements were performed at least in duplicate.

\subsection{Determination of Protein Solubility}

To determine protein solubility, fresh dispersions were centrifuged at $10000 \times \mathrm{g}$ at $20^{\circ} \mathrm{C}$ for $30 \mathrm{~min}$ (Hermle $129 \mathrm{Z} 200 \mathrm{M} / \mathrm{H}$ centrifuge, Hermle Labortechnik; Wehningen, Germany) and then the insoluble debris was separated. Appropriate dilutions of limpid supernatants (100-500-fold) were made with distilled water and protein concentration was determined by the modified Lowry method, in which the increase of copper tartrate concentration allows assay of complex samples containing substantial amount of common interferences such as sucrose and EDTA. ${ }^{[19]}$ Bovine serum albumin was used as standard protein. Protein solubility was expressed as g soluble protein per $100 \mathrm{~g}$ total protein. Assays were performed in triplicate.

\subsection{Optical Microscopy}

Fresh dispersions and emulsions were observed with an optical microscope $(400 \times$ magnification) to analyze their microstructure. An adapted digital camera $(4 \times$ optical zoom; Canon Power Shot A570 IS; Canon Inc., Malaysia) was used to obtain the micrographs. Emulsions were previously diluted $(1: 10 \mathrm{v} / \mathrm{v})$ with distilled water.

\subsection{Determination of Free Calcium Concentration}

To determine free calcium, fresh dispersions were centrifuged at $15000 \times \mathrm{g}$ at $10^{\circ} \mathrm{C}$ for $30 \mathrm{~min}$ (Sigma 3-18KS high-speed centrifuge; 19776-H rotor; Sigma Laborzentrifugen $\mathrm{GmbH}$; Osterode am Harz, Germany) and then the insoluble debris was separated. Afterwards, supernatants were dialyzed against distilled water (1:9 volume ratio) using a dialysis membrane tubing ( $3.5 \mathrm{kDa}$ molecular-weight cut-off). Free calcium was determined on dialysis water by direct colorimetric method with o-cresolphthalein complexone. ${ }^{[20]}$ Assays were carried out in duplicate.

\subsection{Rheological Measurements}

Flow behavior and oscillatory rheology studies were carried out using an AR-G2 rheometer (TA instruments; New Castle, DE, USA). Flow behavior of fresh dispersions was analyzed with a cone-and-plate geometry (gap, $55 \mu \mathrm{m}$; cone diameter, $40 \mathrm{~mm}$; cone angle, $2^{\circ}$ ); shear rate was firstly increased from 0.1 to $100 \mathrm{~s}^{-1}$ for $210 \mathrm{~s}$, then kept constant for $60 \mathrm{~s}$ and finally decreased from 100 to $0.1 \mathrm{~s}^{-1}$ for $210 \mathrm{~s}$. Oscillatory rheology of fresh emulsions was evaluated with a plate-and-plate geometry (gap, $1000 \mu \mathrm{m}$ ); the storage or elastic modulus ( $\mathrm{G}^{\prime}$ ) and the loss or viscous modulus (G") were recorded at an oscillation frequency of $1.0 \mathrm{~Hz}$, within the linear viscoelasticity range (1.0\% strain); then the complex modulus $\left(\mathrm{G}^{*}\right)$ and $\tan \delta$ were calculated as $\left[\left(G^{\prime}\right)^{2}+\left(G^{\prime \prime}\right)^{2}\right]^{1 / 2}$ and $G^{\prime \prime} / G^{\prime}$, respectively. In both experiments the temperature was controlled at $21^{\circ} \mathrm{C}$ with a water bath (Julabo ACW100, Julabo Labortechnik; Seelbach, Germany) associated to the rheometer. Measurements were performed at least in duplicate.

\subsection{Creaming Stability}

Creaming stability of emulsions was evaluated by visual analysis. A sample volume of $10 \mathrm{~mL}$ was incorporated in $10 \mathrm{~mL}$ graduated glass test tubes (in divisions of $0.1 \mathrm{~mL}$ ). The creaming degree (\%) was defined as the volume measured from the bottom of the tube to the inferior limit of the cream phase in percentage relation to the total sample volume. Measurements were performed after storage at $7^{\circ} \mathrm{C}$ for 1,4 , and 7 days.

\subsection{Statistical Analysis}

Data were analyzed by analysis of variance (one-way ANOVA) and test of least significant difference (LSD) using the statistical program Statgraphics Plus 5.1 (Statistical Graphics Corp.; VA, USA). Significance was considered at $p<0.05$. The statistical analysis of rheological data was performed with previous transformation of $\mathrm{G}^{*}$ values into their decimal logarithm.

\section{Results and Discussion}

\subsection{Characterization of Aqueous Dispersions}

The PSDs of DSF aqueous dispersions can be observed in Figure 1, showing the effects of calcium concentration on the microstructure of the systems with different treatments (i.e., at different stages of the obtaining procedure). In these cases, the particles that are able to scatter the light correspond fundamentally to insoluble fibers and aggregated proteins. Dispersions obtained before heating and homogenization showed main populations with modes at $6-8 \mu \mathrm{m}$ and a minor population at $0.8 \mu \mathrm{m}$ (Figure 1a). In these systems, the increasing addition of calcium chloride increased the minor population corresponding to smaller particles; and the $D_{4,3}$ values decreased with increasing concentration of divalent cation (Figure 2a). This result was linked to the binding of calcium by storage soy 

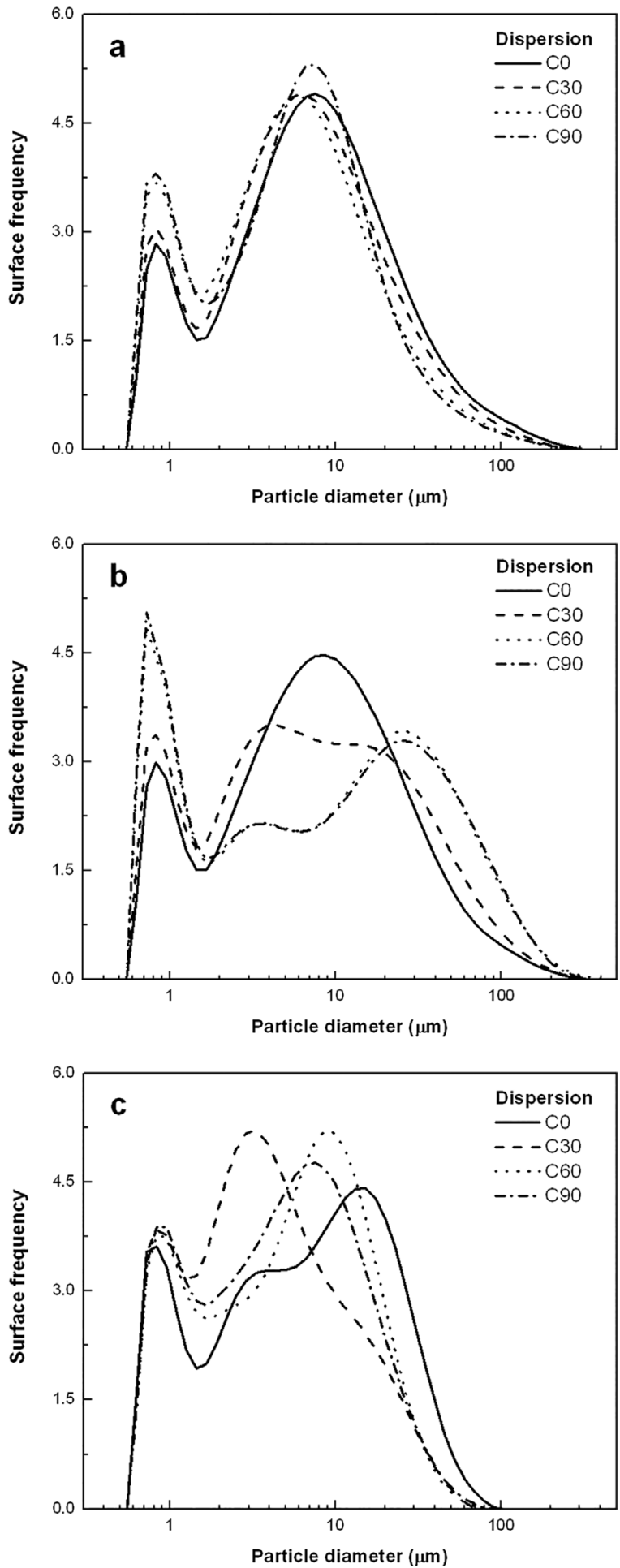

Figure 1. Particle size distribution (expressed as differential surface) of fresh soybean flour dispersions subjected to different treatments (described in Section 2.2) without added calcium (CO) and with calcium addition at 30,60 , and $90 \mathrm{mg} 100 \mathrm{~g}^{-1}$ (C30, C60, and C90, respectively): no heating and no homogenization a); heating and no homogenization b); heating and homogenization c). proteins (glycinin and $\beta$-conglycinin), favoring protein aggregation by electrostatic screening and hydrophobic interactions. ${ }^{[6,8,10]}$ This effect also was demonstrated by the decrease of soluble protein concentration with increasing calcium concentration in dispersions with no heating and no homogenization (Figure 2b). According to optical microscopy, the increase of calcium content in these systems produced a higher concentration of insoluble particles (Figure 3a-d), which can be attributed to higher protein aggregation. The new insoluble particles generated by this calcium-induced protein aggregation were probably smaller than some preexisting particles (e.g., insoluble fibers and bigger aggregates), which would explain the decrease of the $D_{4,3}$ values.

The application of only heating no homogenization produced a different effect, since the heat treatment led to the increase of the $D_{4,3}$ values with increasing calcium concentration until $60 \mathrm{mg} / 100 \mathrm{~g}$ (Figure 2a). The PSDs indicate that this result was attributed to the higher proportion of bigger particles $(>20 \mu \mathrm{m})$ in the systems with added calcium (Figure $1 \mathrm{~b}$ ), probably because of further aggregation by calcium-protein interaction at high temperature. Thus, this effect was enhanced at higher calcium concentrations (60 and $90 \mathrm{mg} / 100 \mathrm{~g}$ ). Moreover, the possible interaction of calcium with insoluble fiber ${ }^{[11]}$ may favor the association of fibers with protein aggregates, where the divalent cation would act as a bridge, increasing the particle size. Indeed, the optical micrographs of heated dispersions show more dense structures at higher calcium content (Figure 3e-h), probably because of a higher interaction between particles. It should be noted that while heating increased the soluble protein concentration in the dispersion $\mathrm{C} 0$, the opposite occurred in the other systems (Figure 2b), indicating that extra insoluble protein was produced by the treatment when the divalent cation was incorporated. These new insoluble particles could be part of the population at $0.8 \mu \mathrm{m}$ and/or be integrated to bigger aggregates. The minimum protein solubility obtained in dispersions C60 and C90 would be given by soy whey proteins, which would remain soluble because they have low sensibility to aggregation by the presence of calcium. This observation is supported by previous works, where the high solubility of proteins isolated from tofu whey, a residual liquid with high calcium content, was effectively observed. ${ }^{[21]}$

The high-pressure homogenization applied after heating decreased the $D_{4,3}$ values of all dispersions, below the original values corresponding to unheated systems (Figure 2a). The calcium-fortified dispersions now presented lower $D_{4,3}$ values than the system $\mathrm{C} 0$, as opposed to the results observed after the heat treatment. The PSDs show that bigger particles were reduced in all cases after homogenization, presenting diameters lower than $100 \mu \mathrm{m}$ (Figure 1c), in comparison to heated, nonhomogenized systems (Figure 1b). Moreover, a higher proportion of bigger particles were observed in the dispersion C0 after homogenization. This last result could be explained by the lower amount of insoluble protein in the system $\mathrm{C} 0$, confirmed by data of soluble protein concentration after homogenization (Figure 2b). The optical micrographs of homogenized dispersions indicate that the addition of calcium led to new insoluble particles apart from the preexisting insoluble fiber (Figure 3i-1). Thus, the dispersion $\mathrm{C} 0$ contained a higher insoluble fiber/ insoluble protein ratio; and this system would have presented a 

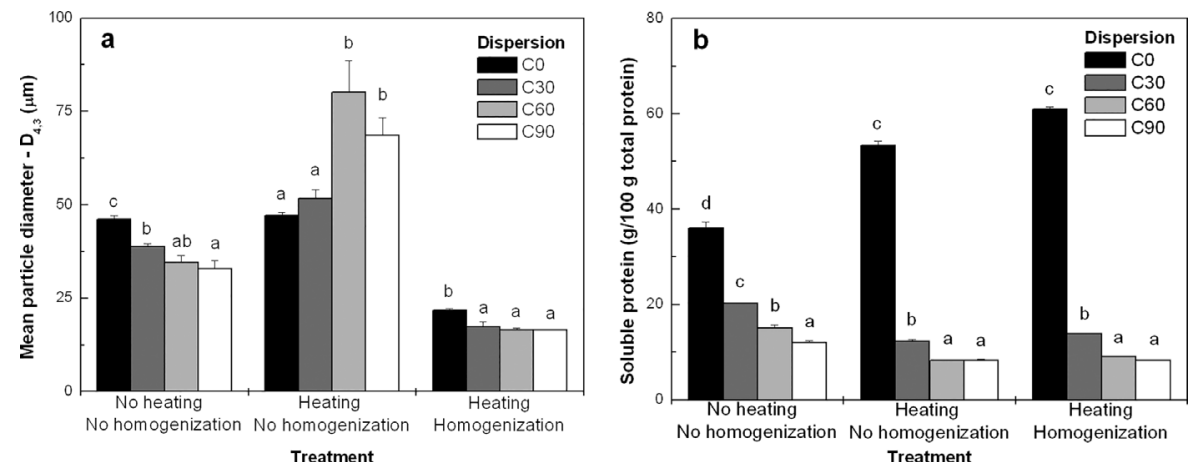

Figure 2. Mean particle diameter $\left(D_{4,3}\right)$ a) and protein solubility values $b$ ) of fresh soybean flour dispersions subjected to different treatments (described in Section 2.2) without added calcium (CO) and with calcium addition at 30,60 , and $90 \mathrm{mg}^{100 \mathrm{~g}^{-1}}$ (C30, $\mathrm{C} 60$, and C90, respectively). Values are means of two replicates and error bars indicate standard deviation. Mean values with different letters indicate significant differences between dispersions with different calcium concentration and same treatment $(p<0.05)$.
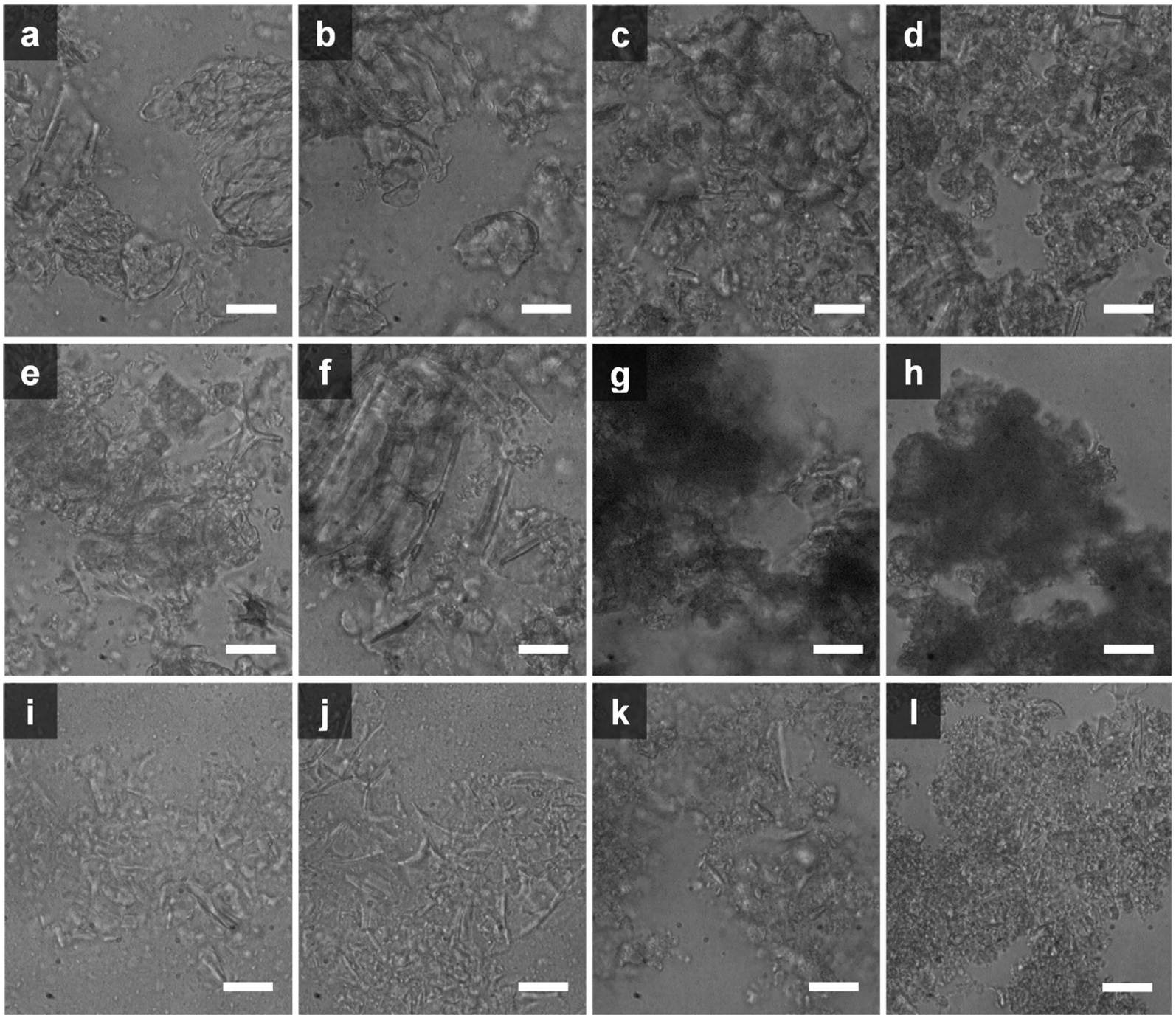

Figure 3. Optical micrographs of fresh soybean flour dispersions subjected to different treatments (described in Section 2.2) without added calcium (CO) and with calcium addition at 30,60 , and $90 \mathrm{mg} 100 \mathrm{~g}^{-1}$ ( $\mathrm{C} 30, \mathrm{C} 60$, and $\mathrm{C} 90$, respectively). No heating and no homogenization: $\mathrm{C} 0 \mathrm{a}$ ); $\mathrm{C} 30 \mathrm{~b}$ ); $\left.\mathrm{C} 60 \mathrm{c}\right)$; ( 90 d). Heating and no homogenization: $(0$ e); $(30$ f); $(60$ g); $(90$ h). Heating and homogenization: $(0$ i); $(30$ j); $(60$ k); $(90$ I). Bar = $20 \mu m$. 
higher proportion of bigger particles because rigid fibers would be less prone to disruption than protein aggregates. However, the dispersions $\mathrm{C} 60$ and $\mathrm{C} 90$ exhibited main populations with higher mode than the system C30 (Figure 1c), probably because a higher calcium concentration produced less breakable aggregates due to the formation of a higher number of crosslinkages. ${ }^{[22]}$ In this context, the determination of free calcium (mg free $\mathrm{Ca} / 100 \mathrm{~g}$ ) on homogenized dispersions gave the following results: $3.26 \pm 0.09,10.03 \pm 0.28,21.18 \pm 2.25$, and $35.97 \pm 0.18$ for C0, C30, C60, and C90, respectively. From these results, and taking into account only the added calcium content, the following linked calcium/insoluble protein ratios (mg linked $\mathrm{Ca} / \mathrm{g}$ insoluble protein) were obtained: $5.61 \pm 0.08,10.33 \pm 0.60$, and $14.25 \pm 0.05$ for $\mathrm{C} 30$, C60, and $\mathrm{C} 90$, respectively. Thus, it can be concluded that a higher calcium amount was bound by proteins at higher concentration of divalent cation, leading to a higher number of cross-linkages. Furthermore, as was also observed by Ono et al., ${ }^{[7]}$ the $\mathrm{pH}$ of dispersions decreased progressively with increasing calcium concentration: $6.60 \pm 0.01,6.03 \pm 0.01,5.83 \pm 0.01$, and $5.60 \pm 0.02$ for $\mathrm{C} 0$, C30, C60, and C90, respectively. This would be another factor producing bigger or less breakable aggregates, because the $\mathrm{pH}$ values at higher calcium concentration were closer to the isoelectric point of storage soy proteins (5.1 and 4.3 for glycinin and $\beta$-conglycinin, respectively). ${ }^{[23]}$

In addition, the flow behavior of homogenized aqueous dispersions with varied calcium concentration is exhibited in Figure 4. After increase and subsequent decrease of shear rate, all dispersions showed a pseudoplastic behavior and hysteresis, ${ }^{[24]}$ giving lower shear stress values at the last stage. These observations indicate that the apparent viscosity of dispersions decreased with increasing shear rate, due to the disruption of structures mainly conformed by aggregated proteins and insoluble fibers. The addition of calcium produced clearly higher shear stress values, attributed to the aggregation of proteins/fibers and the increased volume of insoluble particles

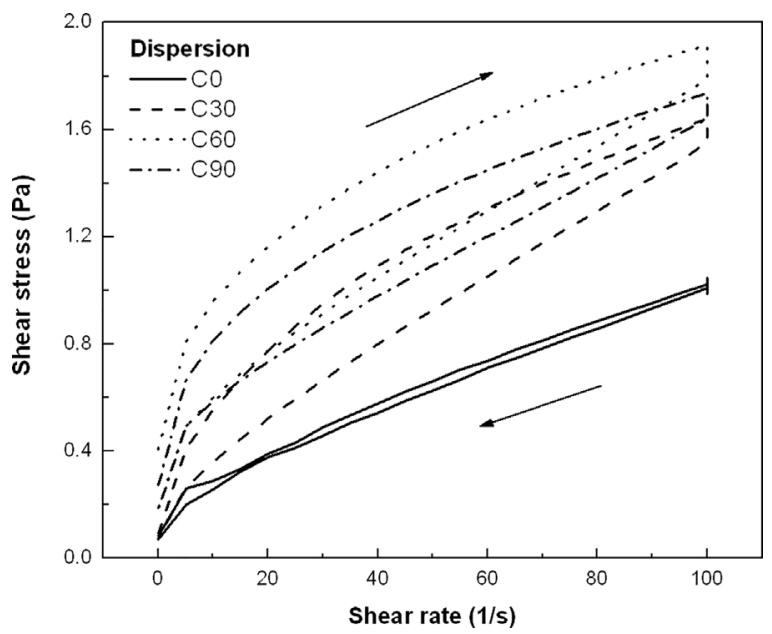

Figure 4. Flow behavior of fresh soybean flour dispersions without added

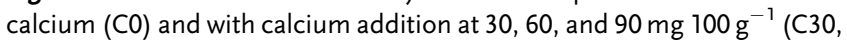
$\mathrm{C} 60$, and $\mathrm{C} 90$, respectively). Dispersions were heated and homogenized as described in Section 2.2.
(Figure 2b). Consequently, the presence of calcium increased the interaction between particles and, thus, increased the apparent viscosity of the systems. ${ }^{[13]}$ Moreover, the divalent cation produced a higher fall of shear stress at constant shear rate $\left(100 \mathrm{~s}^{-1}\right)$ because of the disruption of a higher hydrodynamic volume of structures. However, the highest shear stress levels were observed in the dispersion C60, while the system C90 presented lower values. This result shows that the apparent viscosity of dispersions increased with increasing calcium concentration until certain point and then it decreased with higher addition of divalent cation. The explanation of this last observation could be related to the increase of the linked calcium/insoluble protein ratio with increasing calcium concentration. Previous studies concerned with the microstructure of firm tofu ${ }^{[22]}$ and rheological behavior of soy beverages ${ }^{[17]}$ indicated that an excess of calcium led to more packed protein aggregates due to the production of too many cross-linkages. This adopted structure of protein aggregates would reduce the hydration and interaction of particles, explaining the decrease of apparent viscosity of aqueous dispersions from 60 to $90 \mathrm{mg}$ added $\mathrm{Ca} / 100 \mathrm{~g}$.

\subsection{Microstructure of Emulsions}

The PSDs of emulsions prepared with homogenized dispersions as continuous aqueous phase and sunflower oil as dispersed lipid phase are shown in Figure 5, where the effects of calcium concentration and homogenization method are analyzed. The emulsion HS-0 showed a bimodal distribution with the main population at $7 \mu \mathrm{m}$ (Figure 5a). In this case, the PSD was displaced toward bigger particle diameters when calcium was added and its concentration was increased. Consequently, the $D_{4,3}$ values of emulsions HS increased with increasing calcium concentration (Table 1). This result was attributed to the increased size and flocculation of oil droplets, according to observations by optical microscopy (Figure 6a-d). As it was previously explained, the binding of calcium by soy proteins decreased the soluble protein concentration in the aqueous phase, corresponding to homogenized dispersions (Figure 2b). This would reduce the availability of tensioactive agents to be part of the interfacial film and, thus, the total interfacial area would be decreased and the individual droplets size would be increased. Although some protein aggregates may also be adsorbed at the interface, when the amount of such aggregates increases the interfacial area decreases, because more protein is needed to cover the same surface. ${ }^{[25]}$ Among the calciumfortified systems, the emulsion HS-30 showed lower oil droplets size because a higher proportion of storage soy proteins would stay soluble, maintaining their tensioactive efficiency. In contrast, the emulsions HS-60 and HS-90 would have lower or null quantity of soluble storage proteins; the remaining soluble proteins would be those from soy whey, as it was previously mentioned. This fact would explain the bigger oil droplets observed in the emulsions with higher calcium content. With regard to the flocculation of oil droplets in those systems, it could be related to their higher free calcium content, as it was previously determined on dispersions, and the higher amount of calcium-protein linkages. According to the blob-and-spring 

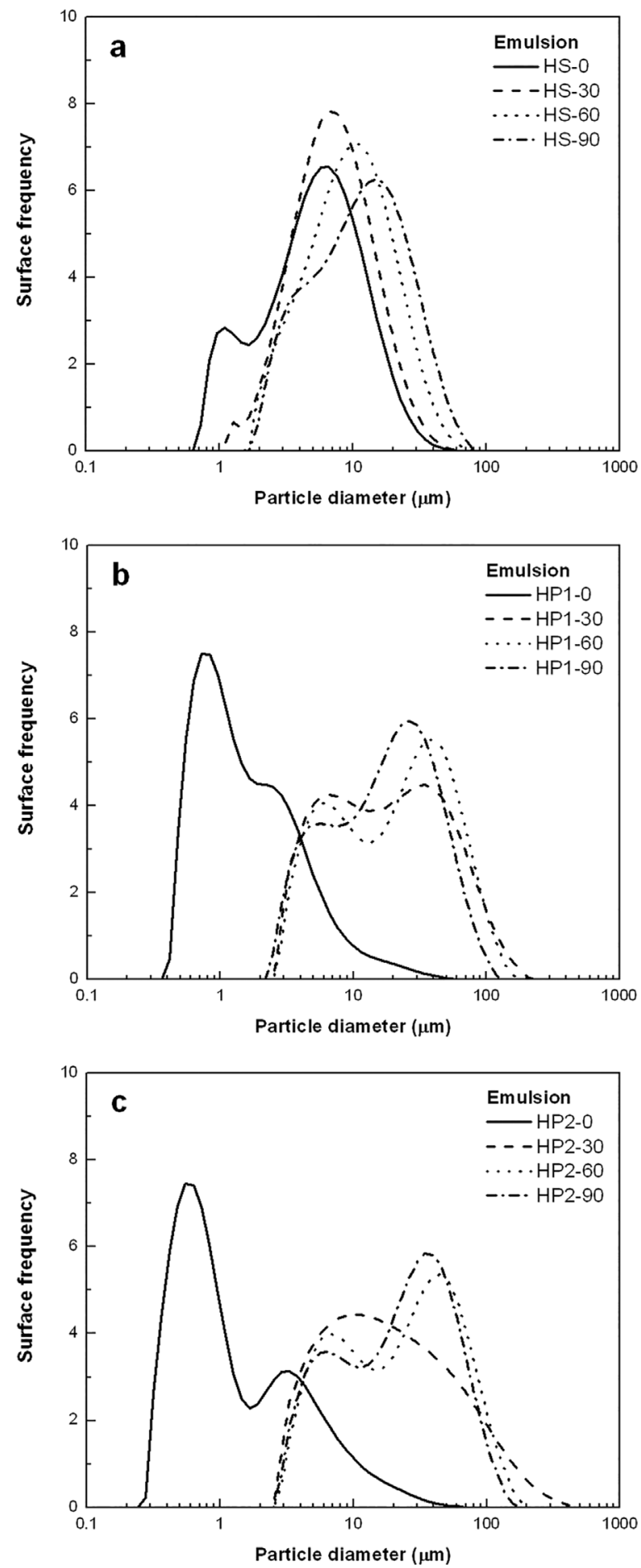

Figure 5. Particle size distribution (expressed as differential surface) of fresh o/w emulsions prepared by mixing of sunflower oil and soybean flour dispersions with different added calcium concentrations. Emulsions were obtained by different homogenization methods (described in Section 2.3): HS a); HP1 b); HP2 c).

model, ${ }^{[26]}$ soluble ions produce thinner protein layers at the interface because they reduce the effectiveness of the repulsion between negatively charged head groups and surface; and binding of positive ions to adsorbed proteins reduces the effective charge of head groups, also leading to thinner layers; consequently, steric repulsion between particles is reduced, inducing aggregation. In this way, the excess of calcium could promote the observed flocculation of oil droplets.

In the absence of calcium, the PSDs of HP1 and HP2 emulsions showed main populations at $0.6-0.8 \mu \mathrm{m}$ (Figure $5 \mathrm{~b}$ and $\mathrm{c}$ ), presenting lower $D_{4,3}$ values than the emulsion HS-0 (Table 1) due to the production of smaller oil droplets as a result of the application of higher homogenization energy ${ }^{[27]}$ This effect can be appreciated by optical microscopy when the emulsions HP1-0 and HP2-0 (Figure 6e and i) are compared to the emulsion HS-0 (Figure 6a). However, the opposite result was observed in the presence of calcium, because at every concentration of divalent cation the employment of higher homogenization energy produced higher $D_{4,3}$ values (HP2 > HP1 > HS; Table 1). The micrographs indicate that this result was attributed to an important aggregation of particles, as it can be observed in emulsions HP1 and HP2 with added calcium (Figure $6 f-h$ and $j-1)$. These big aggregates would be composed by oil droplets, protein aggregates, and even insoluble fibers. Oil droplets may also be joined with each other by bridging flocculation, ${ }^{[28]}$ because the higher homogenization energy produced a higher interfacial area and at the same time the presence of calcium reduced the availability of soluble protein; thus, it is expected that different droplets may be bridged by protein aggregates because the amount of tensioactive agents would be insufficient to cover the interface. This phenomenon would be more pronounced in emulsions HP2 because of the higher applied pressure in comparison to emulsions HP1. Furthermore, the interaction and aggregation of different kinds of particles (oil droplets, protein aggregates, insoluble fibers) would be enhanced at higher homogenization energy due to the generation of higher superficial area and more junction sites, explaining the higher $D_{4,3}$ values of emulsions HP2.

The PSDs of emulsions HP1 and HP2 were also modified by the variation of calcium concentration (Figure $5 b$ and c). In these systems, the biggest particles at the assayed conditions were detected at $30 \mathrm{mgCa} 100 \mathrm{~g}^{-1}$, according to the $D_{4,3}$ values (Table 1). The addition of higher calcium concentrations led to lower $D_{4,3}$ values, indicating that the size of measured aggregates decreased from 30 to $90 \mathrm{mg} \mathrm{Ca} 100 \mathrm{~g}^{-1}$. It should be noted that the size of individual oil droplets in emulsions homogenized at high pressure was increased with the increase of calcium concentration; this was more clearly observed in the optical micrographs of emulsions HP1 (Figure 6f-h). As it was previously stated, this result was related to the decrease of soluble protein in the aqueous phase. Considering this fact, the bigger aggregates measured in the emulsions HP1-30 and HP230 could have different explanations: smaller droplets (i.e., a higher number of droplets) are more prone to aggregation because of the higher collision frequency, aggregates including smaller droplets are able to occlude more continuous aqueous phase within the structure, increasing their effective volume; and the higher the number of droplets the higher the number of junction sites, decreasing the ease of disruption of aggregates at the measurement conditions. ${ }^{[27]}$ In addition, the excess of calcium may lead to more packed and less hydrated structures due to the reduction of steric repulsions, ${ }^{[26]}$ so that aggregation 
Table 1. Mean particle diameter $D_{4,3}(\mu \mathrm{m})$ of fresh o/w emulsions prepared by mixing of sunflower oil and soybean flour dispersions.

\begin{tabular}{lccc}
\hline & \multicolumn{3}{c}{ Homogenization method } \\
\cline { 2 - 4 } $\begin{array}{l}\text { Added calcium } \\
\text { concentration } \\
(\mathrm{mg} / 100 \mathrm{~g} \text { aqueous phase })\end{array}$ & $\mathrm{HS}$ & $\mathrm{HP1}$ & $\mathrm{HP2}$ \\
\hline 0 & $11.97 \pm 0.84 \mathrm{a} \square$ & $8.00 \pm 0.66 \mathrm{a}$ & $9.31 \pm 0.33 \mathrm{a} \bullet$ \\
30 & $12.38 \pm 0.54 \mathrm{a} \square$ & $56.37 \pm 2.77 \mathrm{~d} \bullet$ & $88.68 \pm 2.38 \mathrm{~d} \boldsymbol{\Delta}$ \\
60 & $17.62 \pm 0.88 \mathrm{~b} \square$ & $48.80 \pm 1.13 \mathrm{c}$ & $61.49 \pm 5.85 \mathrm{c} \boldsymbol{\Delta}$ \\
90 & $22.34 \pm 0.48 \mathrm{c} \square$ & $35.88 \pm 1.40 \mathrm{~b}$ & $47.24 \pm 2.03 \mathrm{~b} \boldsymbol{}$ \\
\hline
\end{tabular}

HS, HP1, and HP2 homogenization methods are described in Section 2.3. Values are means of two replicates \pm standard deviation. Mean values with different letters indicate significant differences between emulsions with different calcium concentration and same homogenization method $(p<0.05)$. Mean values with different symbols indicate significant differences between emulsions with different homogenization method and same calcium concentration $(p<0.05)$. of particles would be induced, but the aggregates size would be smaller than those produced by lower calcium concentration.

\subsection{Rheology of Emulsions}

All emulsions HS were liquid regardless the presence of added calcium; and emulsions HP1 and HP2 showed a liquid appearance in the absence of added calcium and a creamy texture when the divalent cation was incorporated. The oscillatory rheology analysis confirmed these appreciations, since emulsions HP1 and HP2 showed higher $\mathrm{G}^{*}$ values than that of HS emulsions at same calcium concentration (Figure 7a). This rheological difference was attributed to the higher interaction between particles in emulsions HP1 and HP2, as a result of the smaller and more numerous oil droplets or the important aggregation of particles produced by high-pressure homogenization. ${ }^{[27,29]}$ Particularly, emulsions HS showed an
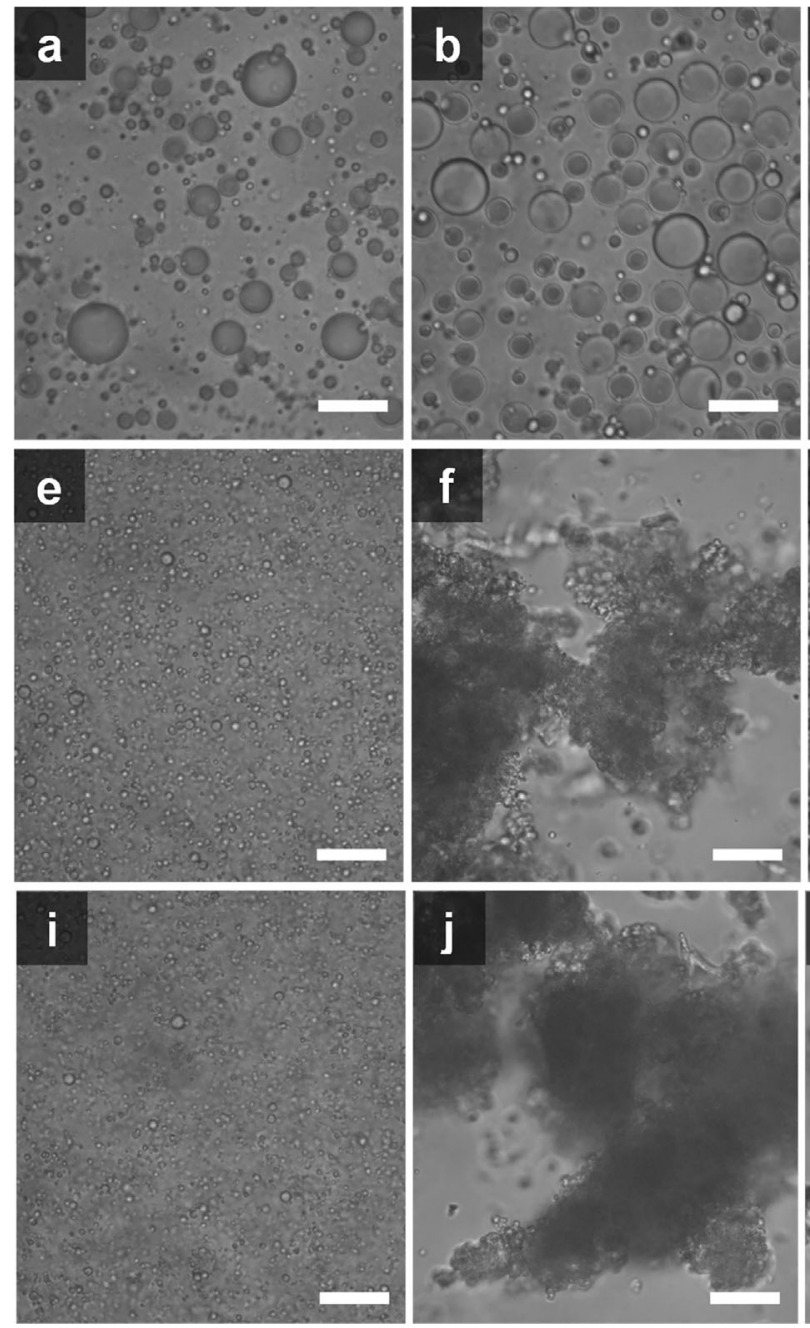
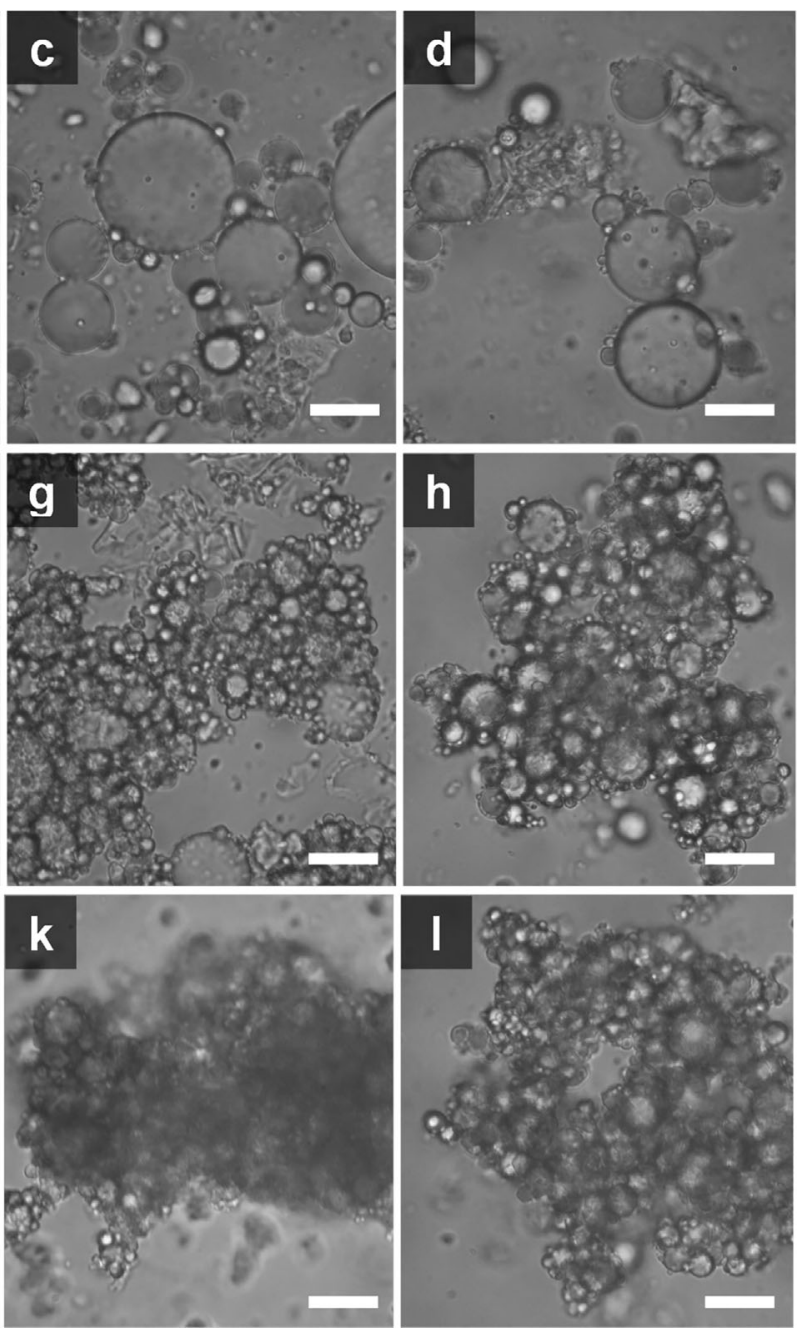

Figure 6. Optical micrographs of fresh o/w emulsions (1:10v/v dilution) prepared by mixing of sunflower oil and soybean flour dispersions with different added calcium concentrations. HS-0 a); HS-30 b); HS-60 c); HS-90 d); HP1-0 e); HP1-30 f); HP1-60 g); HP1-90 h); HP2-0 i); HP2-30 j); HP2-60 k); HP2-90 I). HS, HP1 and HP2 homogenization methods are described in Section 2.3. Bar $=20 \mu \mathrm{m}$. 

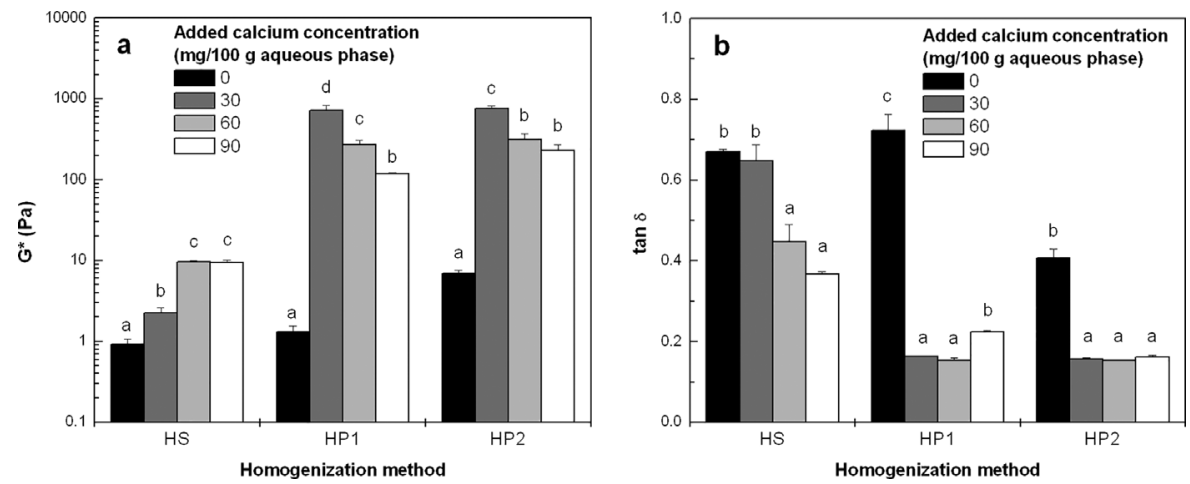

Figure 7. Complex modulus $\left(\mathrm{G}^{*}\right)$ a) and tan $\delta$ values b) of fresh o/w emulsions prepared by mixing of sunflower oil and soybean flour dispersions with different added calcium concentrations. HS, HP1, and HP2 homogenization methods are described in Section 2.3. Values are means of two replicates and error bars indicate standard deviation. Mean values with different letters indicate significant differences between emulsions with different calcium concentration and same homogenization method $(p<0.05)$.

increase of the $\mathrm{G}^{*}$ value with increasing calcium concentration until $60 \mathrm{mg} 100 \mathrm{~g}^{-1}$ despite the increase of oil droplets size. This result would be related to the viscosity of the continuous aqueous phase, which exhibited the highest value at $60 \mathrm{mg} \mathrm{Ca} 100 \mathrm{~g}^{-1}$ according to the previous flow behavior analysis performed on homogenized dispersions (Figure 4); and the flocculation of oil droplets induced by the excess of calcium (Figure $6 c$ and d) may also have contributed to the increase of the $G^{*}$ value. With regard to emulsions HP1 and HP2, the presence of calcium produced clearly higher $\mathrm{G}^{*}$ values than the corresponding controls (Figure $7 \mathrm{a}$ ) due to the aggregation of particles induced by the divalent cation (Figure 6e-1). In a previous work on $\mathrm{o} / \mathrm{w}$ emulsions stabilized with soy protein isolate, it has been stated that when oil droplets are bridged by protein aggregates the viscosity of the system increases, ${ }^{[30]}$ as it would also occur in emulsions HP1 and HP2 in the presence of calcium. Nevertheless, the increase of calcium concentration from 30

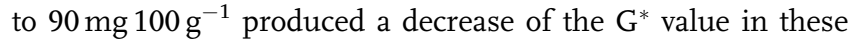
systems. The explanation of this result would be linked to the lower aggregation degree in emulsions HP1 and HP2 with higher calcium concentration (Table 1).

The rheological study of emulsions was completed with the analysis of the $\tan \delta$ values (Figure $7 \mathrm{~b}$ ). All systems showed $\tan \delta$ values below 1 , indicating a more elastic than viscous character $\left(G^{\prime}>G^{\prime}\right)$. The emulsions HS-60 and HS-90 evidenced lower tan $\delta$ values than the systems with same homogenization method and lower calcium content. This result could be attributed to the higher volume and interaction of insoluble particles and the flocculation of oil droplets produced by the divalent cation, enhancing the elastic character of the system. With respect to emulsions HP1 and HP2, the tan $\delta$ values were always lower in the presence than in the absence of added calcium, probably because the aggregation of particles allowed the retention of water within the structure, producing gel-like systems with higher elastic character. Most emulsions HP1 and HP2 with added calcium showed a similar result, indicating that at certain aggregation degree the systems reached a minimum $\tan \delta$ value. Only the emulsion HP1-90 presented a higher $\tan \delta$ value than that minimum, which may be linked to its lower $D_{4,3}$ value (i.e., smaller or less hydrated aggregates) in comparison to the other calcium-fortified systems homogenized at high pressure (Table 1).

\subsection{Quiescent Stability of Emulsions}

The creaming stability results are shown in Table 2 . Initially, all emulsions exhibited a homogeneous appearance. Among the liquid emulsions HS, the system HS-0 evidenced the highest creaming degree, while the addition of calcium prevented or reduced the ascending movement of the dispersed lipid phase. According to Stokes' law, the creaming rate $(v)$ of an isolated spherical particle in a liquid is defined by the following

Table 2. Creaming degree (\%) of o/w emulsions prepared by mixing of sunflower oil and soybean flour dispersions without added calcium and with addition of calcium at different concentrations.

\begin{tabular}{lccc}
\hline & \multicolumn{3}{c}{ Storage time (days) } \\
\cline { 2 - 4 } Emulsion & 1 & 4 & 7 \\
\hline HS-0 & $34 \pm 6$ & $36 \pm 6$ & $36 \pm 6$ \\
HS-30 & Stable & Stable & Stable \\
HS-60 & $13 \pm 2$ & $17 \pm 3$ & $18 \pm 3$ \\
HS-90 & $14 \pm 2$ & $16 \pm 3$ & $17 \pm 3$ \\
HP1-0 & Stable & Stable & Stable \\
HP1-30 & Stable & Stable & Stable \\
HP1-60 & $11 \pm 1$ & $12 \pm 1$ & $13 \pm 2$ \\
HP1-90 & Coagulated & Coagulated & Coagulated \\
HP2-0 & Stable & Stable & Stable \\
HP2-30 & Stable & Stable & Stable \\
HP2-60 & $10 \pm 1$ & $10 \pm 1$ & $10 \pm 1$ \\
HP2-90 & Coagulated & Coagulated & Coagulated \\
\hline
\end{tabular}

HS, HP1, and HP2 homogenization methods are described in Section 2.3. Emulsion nomenclature is described in Section 2.3. Values are means of two replicates \pm standard deviation. "Stable" denotes that no creaming was observed. "Coagulated" indicates that a heterogeneous appearance was observed. 
equation $^{[27]}$ :

$v=\frac{2 g r^{2}\left(\rho_{2}-\rho_{1}\right)}{9 \eta_{1}}$

where $r$ is the radius of the particle, $g$ is the acceleration due to gravity, $\rho_{1}$ and $\rho_{2}$ are the densities of the continuous and dispersed phases, respectively, and $\eta_{1}$ is the shear viscosity of the continuous phase. Thus, the higher creaming stability in emulsions HS with added calcium can be explained by the higher apparent viscosity of the continuous aqueous phase (Figure 4) produced by the presence of the divalent cation. It should be noted that no creaming was observed in the emulsion HS-30 after cold storage for 7 days, but the systems with higher calcium contents did show gravitational separation. The latter result was attributed to the increased particles size of emulsions HS-60 and HS-90 (Table 1 ) as a result of bigger and flocculated oil droplets (Figure $6 c$ and d), which increases creaming rate according to Equation (1).

Emulsions HP1-0 and HP2-0 were liquid and evidenced absence of creaming (Table 2). The high stability of these systems can also be explained by Equation (1), due to the lower oil droplets size produced by high-pressure homogenization (Figure 6e and i) in comparison to high-speed homogenization (Figure 6a). Moreover, emulsions HP1-30 and HP2-30 did not show gravitational separation, but the systems HP1-60 and HP260 presented slight destabilization in spite of their creamy texture, manifested by high $\mathrm{G}^{*}$ values (Figure 7a). This observation could be related to the size and structure of aggregates, because the aggregation of particles can prevent the ascending movement of the dispersed phase due to the formation of a three-dimensional network and the retention of water within the structure; but it can increase the creaming rate when the interaction and hydration of aggregates are low. ${ }^{[31]}$ According to the microstructure and rheological results, the highest $D_{4,3}$ and $\mathrm{G}^{*}$ values in emulsions HP1 and HP2 were detected at $30 \mathrm{mgCa} 100 \mathrm{~g}^{-1}$ (Table 1; Figure 7a), alleged to higher hydration and interaction of aggregates. These characteristics may increase the global density of the structure and favor the formation of a network, explaining the absence of creaming in these systems. On the other side, the smaller and more packed aggregates in emulsions HP1-60 and HP2-60 would have insufficient water retention capacity to avoid phase separation. It should be mentioned that all emulsions presented slightly higher $D_{4,3}$ values after cold storage for 7 days in comparison to initial data, indicating further aggregation of particles and/or coalescence of oil droplets, but the systems showed the same relative order of particle sizes (data not shown). With respect to emulsions HP1-90 and HP2-90, their creaming degree was not measured because these systems exhibited a heterogeneous appearance after cold storage for 1 day, probably due to the exudation of water as a consequence of the coagulation of proteins at excessive calcium concentration combined to the relatively low water retention of aggregates.

\section{Conclusions}

This work showed that it is possible to prepare DSF dispersions with proteins being denatured and aggregated by heating in the presence of calcium, employing high-energy homogenization devices. These dispersions can be used for the elaboration of $\mathrm{o} / \mathrm{w}$ emulsions, since the aqueous systems presented adequate emulsifying and stabilizing capacities at certain conditions. The obtained emulsions showed varied characteristics regarding their microstructure, rheology, and stability, as a result of the combined variation of calcium concentration and homogenization energy. Emulsions prepared with moderate calcium content by high-pressure homogenization reached the highest particle aggregation degree, viscosity and creaming stability, attributed to the formation of a hydrated network integrating flocculated oil droplets, protein aggregates, and fibers. In this way, calcium would not only contribute a nutritional benefit, but it could also be used as a functional ingredient. For instance, the formulation of vegetable food emulsions simulating the texture of whipped dairy cream could be accomplished, because the aggregation of fat globules by partial coalescence would be replaced by the aggregation of particles due to the presence of calcium.

\section{Abbreviations}

DSF, defatted soybean flour; $\mathrm{HP1}$, high pressure at $10 \mathrm{MPa}$; HP2, high pressure at $20 \mathrm{MPa}$; $\mathrm{HS}$, high speed; PSD, particle size distribution.

\section{Acknowledgments}

Authors wish to acknowledge the financial support of Consejo Nacional de Investigaciones Científicas y Técnicas (CONICET; PIP 2012-2014 No. 11220110100398), Universidad Nacional de Quilmes (53/1037 I+D grant), and Agencia Nacional de Promoción Científica y Tecnológica (FONCyT, PICT-2016-2699). A. L. Márquez, J. R. Wagner, and G. G. Palazolo are researchers of CONICET.

\section{Conflict of Interest}

The authors declare no conflict of interest.

\section{Keywords}

calcium, defatted soybean flour, emulsions, high-pressure homogenization, high-speed homogenization

Received: November 29, 2017

Revised: March 23, 2018

Published online: April 24, 2018

[1] K. Yasumatsu, K. Sawada, S. Moritaka, M. Misaki, J. Toda, T. Wada, K. Ishii, Agric. Biol. Chem. 1972, 36, 719.

[2] V. Kumar, A. K. Sinha, H. P. S. Makkar, G. De Boeck, K. Becker, Crit. Rev. Food Sci. Nutr. 2012, 52, 899.

[3] A. Liutkevičius, V. Speičienè, A. Kaminskas, V. Jablonskienè, G. Alenčikienė, A. Mieželienė, L. Bagdonaitè, D. Vitkus, G. Garmiené, CYTA - J. Food 2016, 14, 309.

[4] J. Krongsin, C. Gamonpilas, P. Methacanon, A. Panya, S. M. Goh, Food Hydrocolloid. 2015, 50, 128. 
[5] P. Chaiwanon, P. Puwastien, A. Nitithamyong, P. P. Sirichakwal, J. Food Compos. Anal. 2000, 13, 319.

[6] A. G. Appu Rao, M. S. Narasinga Rao, Cereal Chem. 1975, $52,21$.

[7] T. Ono, S. Katho, K. Mothizuki, Biosci. Biotech. Biochem. 1993, 57, 24.

[8] P. Pathomrungsiyounggul, A. S. Grandison, M. J. Lewis, J. Food Sci. 2007, 72, 428.

[9] J. M. Whittinghill, J. Norton, A. Proctor, J. Am. Oil Chem. Soc. 2000, 77, 37.

[10] L. S. Canabady-Rochelle, M. Mellema, Colloids Surf. A 2010, 366, 110.

[11] L. Corneau, C. Lavigne, J. A. Zee, T. Desrosiers, Nutr. Res. 1996, 16, 1659.

[12] S. Chen, Aceites y Grasas 2006, 63, 238.

[13] P. Pathomrungsiyounggul, M. J. Lewis, A. S. Grandison, Food Chem. 2010, 118, 808.

[14] F. Yazici, V. B. Alvarez, M. E. Mangino, P. M. T. Hansen, J. Food Sci. 1997, 62, 535.

[15] Q. Zhang, L. Wei, F. Meiqin, M. Dong, Food Hydrocolloid. 2013, 33, 106.

[16] A. L. Márquez, J. R. Wagner, J. Texture Stud. 2010, 41, 651.

[17] A. L. Márquez, G. N Salvatore, R. G. Otero, J. R. Wagner, G. G. Palazolo, LWT - Food Sci. Technol. 2015, 62, 474.
[18] P. A. Sobral, G. G. Palazolo, J. R. Wagner, Aceites y Grasas 2012 , $87,82$.

[19] M. A. K. Markwell, S. Haas, L. L. Bieber, N. E. Tolbert, Anal. Biochem. 1978, 87, 206

[20] L. G. Morin, Am. J. Clin. Pathol. 1974, 61, 114.

[21] P. A. Sobral, G. G. Palazolo, J. R. Wagner, J. Agric. Food Chem. 2010, $58,10092$.

[22] F.-J. Kao, N.-W. Su, M.-H. Lee, J. Agric. Food Chem. 2003, 51, 6211.

[23] Y. Yuan, Z.-L. Wan, X.-Q. Yang, S.-W. Yin, Food Res. Int. 2014, 55, 207.

[24] J. R. Wagner, D. A. Sorgentini, M. C. Añón, J. Agric. Food Chem. 1992, 40, 1930.

[25] K. Nishinari, Y. Fang, S. Guo, G. O. Phillips, Food Hydrocolloid. 2014, 39, 301.

[26] D. S. Horne, J. Leaver, Food Hydrocolloid. 1995, 9, 91.

[27] D. J. McClements, Food Emulsions: Principles, Practice and Techniques. CRC Press, New York 1999.

[28] E. Dickinson, F. O. Flint, J. A. Hunt, Food Hydrocolloid. 1989, 3, 389.

[29] A. L. Márquez, J. R. Wagner, J. Am. Oil Chem. Soc. 2012, 89, 1857.

[30] M. Keerati-u-rai, M. Corredig, Food Hydrocolloid. 2009, 23, 2141.

[31] V. J. Pinfield, E. Dickinson, M. J. W. Povey, J. Colloid Interface Sci. 1997, 186,80 